\title{
Artificial neural networks applications in building energy predictions and a case study for tropical climates
}

\author{
Melek Yalcintas ${ }^{1, *, \dagger}$ and Sedat Akkurt ${ }^{2, *}$ \\ ${ }^{1}$ Amel Technologies, Inc., 1164 Bishop St. Suite 124-302, Honolulu, HI 96813, U.S.A. \\ ${ }^{2}$ Department of Mechanical Engineering, Izmir Institute of Technology, Urla, 35430 Izmir, Turkey
}

\begin{abstract}
SUMMARY
This study presents artificial neural network (ANN) methods in building energy use predictions. Applications of the ANN methods in energy audits and energy savings predictions due to building retrofits are emphasized. A generalized ANN model that can be applied to any building type with minor modifications would be a very useful tool for building engineers. ANN methods offer faster learning time, simplicity in analysis and adaptability to seasonal climate variations and changes in the building's energy use when compared to other statistical and simulation models. The model herein is presented for predicting chiller plant energy use in tropical climates with small seasonal and daily variations. It was successfully created based on both climatic and chiller data. The average absolute training error for the model was $9.7 \%$ while the testing error was $10.0 \%$. This indicates that the model can successfully predict the particular chiller energy consumption in a tropical climate. Copyright (C) 2005 John Wiley \& Sons, Ltd.
\end{abstract}

KEY WORDS: artificial neural network; energy use; building; climate

\section{INTRODUCTION}

Current statistics of energy use in different sectors show that the building sector uses approximately 40 per cent of the world's electricity. This electricity is used for heating, air conditioning and ventilation (HVAC), lighting, operation of various equipment including computers, processors and household related equipment, etc. However, power usage is often inefficient with regard to the overall building operability. The main reasons for this inefficiency are poor building envelope design, oversized HVAC equipment, inefficient HVAC and lighting controls and maintenance related issues.

As fossil fuel based energy resources decrease and energy demand increases, better efficiency of energy use and the search for alternative energy resources require immediate attention. Also environment related concerns, such as ozone layer depletion and increase in the carbon dioxide level in the air, necessitate a greater priority for this attention. This paper focuses on the

\footnotetext{
*Correspondence to: Melek Yalcintas, Amel Technologies, Inc., 1164 Bishop St. Suite 124-302, Honolulu, HI 96813, Hawaii, U.S.A.

†E-mail: melekyalcintas@hawaii.rr.com

E-mail: sedatakkurt@iyte.edu.tr
} 
efficiency aspects of building energy use. More specifically, the development of building energy savings methods/models is emphasized. Measurements of the current energy consumption for a particular building would serve as primary input for the methods/models in order to predict future energy consumption. Such a prediction would be useful in several ways including: building health monitoring and diagnostics, equipment controls, energy demand estimation by utility companies, and energy savings predictions due to equipment retrofits or implementation of an energy conservation measure (ECM).

A common practice amongst facility managements is a periodic review of total building energy consumption or of a specific equipment performance. As a result of the energy consumption reviews, certain ECMs are evaluated that would reduce the building's energy use while maintaining its efficiency. Typical ECMs evaluated in such studies are: building cooling equipment and air distribution system evaluation and retrofits, building envelope, facility management system (FMS) retrofits and lighting retrofits. The building energy analysis is done under preliminary energy assessments (PEA) or Energy Feasibility Studies, sponsored by utility companies or facility managements. Usually, the PEA is a simpler analysis based on information gathered by walkthrough surveys and spot measurements. Yearly building electricity consumption is estimated based on building schedule and seasonal diversities.

Moreover, Energy Feasibility Studies offer still more detailed analysis when compared to the PEAs. This is so even when using the general assumptions and analysis methods similar to the PEA. In addition to the data collected for the PEA analysis, a building simulation model is developed and energy use is measured for longer periods of time. While the feasibility studies are more effective when compared to the PEAs, they have several shortcomings in the analysis. To begin with, the building simulation model may not reflect the actual building performance. The main variables considered in the building simulation model are: building envelope, building occupancy rate, lighting, HVAC equipment and ventilation rate. In practice, usually the exact measurements for variables in the simulation models are not readily available. Instead, the best estimates are applied to the model, and the variables are scaled or modified to match the simulation results with the actual measurements. When there is an appearance that the simulation results may agree with the measurements, still they may not reflect the correct ECM savings. Secondly, linear regression methods are widely used to approximate the trends in the measured data and often can be inaccurate. The developed linear regression models usually present the approximations with a relatively high degree of error when compared to other potential analysis methods such as artificial neural networks (ANN).

These aforementioned limitations, associated with the currently used energy assessment methods, necessitate improved alternative tools/techniques. Artificial neural networks, representing non-parametric techniques for achieving arbitrarily complex functional mappings, are promising for wide applications in whole-building energy predictions; and that includes ECM savings as well as FMS controls. Additional advantages of ANN methods over other techniques, such as statistical and simulation methods, are their faster learning time, simplicity in analysis and adaptability to changes in a building's energy use.

\section{ARTIFICIAL NEURAL NETWORK CONCEPT}

The computational structure of ANN consists of an input layer, which accepts patterns from the environment, an output layer that shows response with regard to the environmental variables, 
and hidden layers. The hidden layers which do not directly interact with the environment, however, enact the primary function of relating the input to the output. These layers consist of input weights, biases and transfer functions.

The neural network training process simply involves modification of weights until the predicted output is in close agreement with the actual output. Defined relations between the input layers, the hidden layers and the output layers determine a particular neural network model. Three types of networks used most commonly in ANN applications are feedforward networks, competitive networks and recurrent associative memory networks. Furthermore, each network type may have different learning rules. The learning rules are described in broad categories of supervised learning, unsupervised learning and reinforcement (or graded) learning rules. Many studies on ANN theory have been published along with the development of the ANN method. Zurada (1992) presents the theory of Neural Networks which can be followed by readers with different technical trainings. A practical description of ANN methods with sample applications is presented in Hagan et al. (1997).

\section{ANN APPLICATIONS IN BUILDING INDUSTRY}

The ANN has been investigated for its applicability in building energy predictions over the past ten years (Ansett and Kreider, 1993; Curtiss et al., 1993; Cohen and Krarti, 1995; Kreider et al., 1995; Haberl and Thamilseran, 1996; Breekweg et al., 2000). Various neural network architectures have been applied in energy predictions. They include backpropagation, recurrent neural networks, autoassociative neural networks and general regression neural network demonstrating relatively successful results having coefficient of variations in the range of 2-40\% (Ansett and Kreider, 1993; Curtiss et al., 1993; Cohen and Krarti, 1995; Kreider et al., 1995; Haberl and Thamilseran, 1996; Breekweg et al., 2000). These variations in the accuracy of the predictions depend mostly on the ANN architecture used, the regularity of the building operation and the accuracy of data measurement devices.

More specifically, in a study by Ansett and Kreider (1993), building utility measurement data from a university campus centre, including electricity, natural gas, water and steam use, were modelled. The study considered weather, building occupancy and activity as the independent variables. Backpropagation architecture was used in this effort. The main focus was on testing different training methods, layering and data input order. The study presented encouraging potential for the application of neural networks in building energy modelling. The study also stated the need for future investigation in selecting more accurate and effective learning algorithms.

Curtiss et al. (1993) used ANN to optimize energy consumption on an HVAC system. In this approach, the weather and building occupancy were considered as independent variables, and the HVAC system setpoints such as mixed air temperature, chilled water temperature, duct static pressure and chilled water flow rate were considered as dependent variables. Optimum setpoints were identified by varying the dependent variables that would yield the minimum electricity consumption. The building data were generated by using an HVAC Laboratory. The results of this study showed the need to apply the model to larger sized buildings with actual building measurement data, in order to validate the ANN method's efficiency.

Cohen and Krarti (1995) used energy consumption data generated from the DOE-2.1E Building Energy Analysis Program as input to the ANN model developed. The model was based 
on multi-layered feedforward networks. This study mentioned the potential use of ANN methods in building energy savings estimates and recommended that future ANN modelling studies be done based on 'real' building measurement data.

Kreider et al. (1995) investigated the prediction of future building energy consumption and system identification without the knowledge of immediate past energy consumption. Recurrent neural networks were used in the modelling. According to the authors, the recurrent networks offer an accurate method for predicting hourly energy use well into the future for thermal end uses when only weather data are known. During network training, actual measured data from a few past hours were used as input to the model. However, during the prediction period, the network's own outputs were cycled back into the inputs. The building energy data for this model were also generated from the DOE-2.1E Building Energy Analysis Program. Although the error rate was relatively higher in this method when compared to, for example, the backpropagation method, it was still presented as an applicable method in predicting the future building energy use for retrofit energy savings estimation purposes. This study also stated the need for future study based on 'real' building measurement data.

As part of an energy predictor competition titled 'Great Energy Predictor Shootout', Chonan et al. (1996) applied Bayesian neural network for estimating building energy use. In this method, the known relationship between the input variables and output was used in combination with the neural network training. Jang et al. (1996) used an auto-associative neural network in predicting missing building input-output data based on feedforward network identity mapping. This method is effectively used when the building data have been available for some periods of time and missing for other periods of time. The noise filter capabilities of auto-associative neural networks proved to be effective in preprocessing the model data.

In another study, Curtiss (1996) described the use of neural networks in continuous control of feedback loops in an HVAC system and overall building energy use prediction. In this method, the input and output training data set were updated with new input data and a neural network output prediction from one previous time segment. The training data set was renewed with the latest building information and kept current for the near future predictions. Additionally, in this study, Curtiss used the neural network control algorithm along with the traditional PI control algorithm to develop the optimum control parameters and enhance control capabilities of both methods.

Breekweg et al. (2000) evaluated a number of ANN techniques in the development of a generalized method for building energy-related fault detection. Real-time data from four different buildings and simulation data from one building were modelled based on normalized radial basis function ( $\mathrm{RBF}$ ), specifically the general regression neural network (GRNN) as the normalized RBF was used. The coefficient of variation was higher, in the range of $20-40 \%$ for most buildings, except two buildings, which were in the range of $4-8 \%$. The large deviations in the results were attributed to the quality of data measurement, building operation consistency and minimization of the noise elements in the data set. This study also reported the necessity to test the developed ANN model with energy data from different buildings in order to ensure the generalizing capacity of the model.

Additionally, a literature search was conducted for building energy use prediction models developed for tropical climates. However, to the authors' knowledge, no specific study was found on the topic.

Artificial neural networks have successfully passed the research stages and found real time applications in many technologies including aerospace, defense, automotive, manufacturing process controls, etc. However, such research development and acceptance of ANN as a useful 
tool in the building and utility sector has not been accepted yet. This is mainly because (1) there is an absence of building automation systems, (2) there is high cost associated with installation of continuous measurement devices, (3) buildings have a longer life period, which limits the prompt adaptation of new technologies. Since the current concerns with decreasing energy resources and a better commitment to have healthy building environments are becoming more and more a priority, the development of building technology for optimum energy performance is unavoidable.

The up to date research studies on the ANN applications in building energy predictions have shown promising results; yet further research is needed to address several issues:

(i) determining the most suitable ANN architecture in building energy use applications;

(ii) developing methods to deal with noise in the data;

(iii) developing generalized ANN architectures which can be applied for energy predictions of different building types with minimum modifications in the ANN structure;

(iv) targeting the coefficient of variations as low as $5 \%$ with consistency;

(v) identifying new applications in the HVAC field such as controls.

In addition, the ANN models in the work cited here have used building energy data from building simulation, laboratory experiments and actual building measurement data. While for the sake of simplicity the simulation data in the initial ANN modelling stages are useful, it is essential to use actual building data during the later development stages to account for the possible imperfections in the measured data. Also, the actual building data are the best indicator of the building features, operation and equipment efficiency. However, as mentioned earlier, the noise in the measurement data also has to be dealt with when employing actual measurements in the ANN modelling. Therefore, repeated building data measurements from different buildings should be used in developing the ANN model.

\section{AN ANN BUILDING CASE STUDY FOR TROPICAL CLIMATES}

A 42 storey commercial building with approximately $41800 \mathrm{~m}^{2}$ space in downtown Honolulu, Hawaii was selected for a case study for ANN building energy prediction. The basement housed the chiller room, a mechanical pump room, building maintenance offices, and a parking garage. The plaza level first floor and second floor contained the entry lobby restaurants and retail offices, and additional parking garages. Parking garage spaces took up 5-12 floors. The 14th floor and the upper levels of the building are separated into two towers: an office tower and a residential condominium tower. The 14th floor also contains a recreational deck with a residential lounge and a pool. The cooling towers, exhaust fans and some other mechanical elevator equipment are located on the roof of the residential tower. The building is air conditioned by a central chilled water plant consisting of three chillers with a total 1250 ton capacity. Air conditioning in the office tower is provided for $13-15 \mathrm{~h}$ during the day, and air conditioning for the residential tower is provided $24 \mathrm{~h}$ a day which is controlled by thermostats in each residential unit. Floor air handlers circulate the conditioned air through variable air volume (VAV) terminal units.

This multiple utility building requires the building equipment to operate ' $24 / 7$ ' and has a building automation system (BAS). The chiller electricity use, chilled water flow rate, chilled 
water supply and return temperatures and air handling unit electricity use is monitored continuously. For this study, which was done over a period of three weeks, the hourly chilled water flow rate, chilled water supply and return temperatures, building occupancy rate, and hourly local climate data were used in predicting the total chiller power by the ANN method.

This particular building presented two unique considerations. Firstly, it is located in the tropical climate of Honolulu, Hawaii where variations between the day and night, and summer and winter are minimal. In summer, the maximum dry bulb temperature average for Honolulu is $31.1^{\circ} \mathrm{C}$ and the minimum dry bulb temperature average is $24.4^{\circ} \mathrm{C}$. The average wet bulb temperature is $22.8^{\circ} \mathrm{C}$. In winter, the maximum dry bulb temperature average is $27.2^{\circ} \mathrm{C}$ and the minimum dry bulb temperature average is $19.5^{\circ} \mathrm{C}$. The average wet bulb temperature is $18.9^{\circ} \mathrm{C}$. Average wind velocity in both summer and winter is relatively consistent at $10 \mathrm{mph}$. In this climate, air conditioning is required during the day, through the whole year and during the night, most of the time.

Secondly, the building houses a variety of functions including office, residential, restaurants and recreation. All of these have different air conditioning requirements and schedules, while energy use throughout the day and night is continuous. The small variations in the seasonal weather conditions and continuous building use presents consistent data for the ANN analysis and this in turn gives a better prediction capacity for the developed ANN energy model.

\section{ANN MODEL INPUT AND OUTPUT DATA}

In this study, the power consumption of the central chiller plant, including the chillers, cooling tower and pumps, was modelled based on the ANN method. The data used in the model cover the time period from 4 April 2001 to 16 April 2001. Figure 1 shows the chiller plant power consumption trend for this time period. Independent input variables mainly consisted of climate data, and the model output was the chiller plant power consumption. Hourly climate data were obtained from the National Climate Data Center for April 2001. The climate data variables considered were specifically: dry bulb temperature, wet bulb temperature, dew point temperature, relative humidity percentage, wind speed and wind direction. Table I lists the input and output variables used in the model construction. Unlike the weather data, the data for hourly power consumption of the chiller plant were not available for every hour of the $24 \mathrm{~h}$ a day. Therefore, a matching of the weather and chiller power data produced a total of 121 data sets to be used for the model creation. This was less than the total number of possible combinations of 312 for 13 days.

\section{MODEL DEVELOPMENT}

ANNs contain layers of processing elements. Training of the ANN model is carried out by feeding normalized data (all input quantities are compressed to a common scale of 0.1 and 0.9 ) to the leftmost input layer neurons as shown in Figure 2 when a backpropagation (BP) algorithm is employed. Normalization of input data is accomplished via the following formula

$$
x_{i}=0.1+0.9\left(x_{i}-x_{\min _{i}}\right) /\left(x_{\max _{i}}-x_{\min _{i}}\right)
$$


Total Chiller Power Consumption, 4/04/2001, 4/16/2001
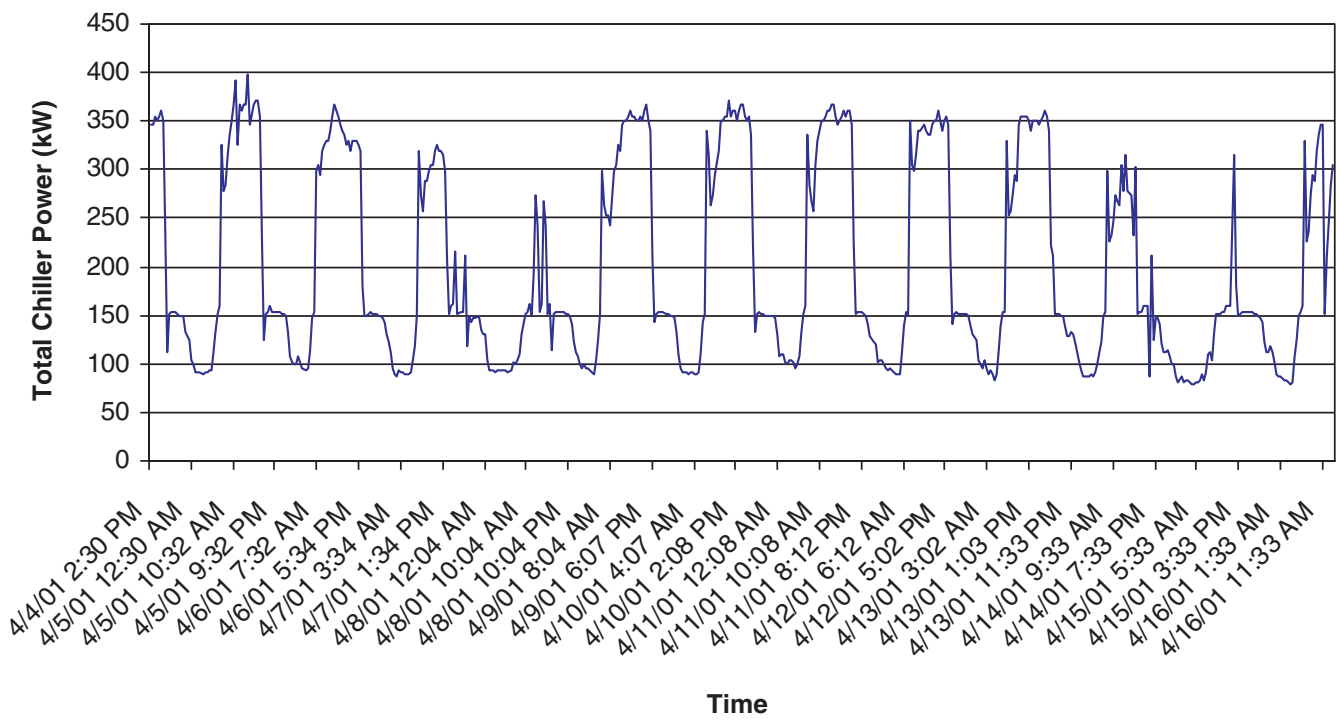

Figure 1. Total chiller plant power consumption from 4 April 2001 to 16 April 2001.

Table I. The data used in ANN model construction.

\begin{tabular}{lcc}
\hline & \multicolumn{2}{c}{ ANN model data } \\
\cline { 2 - 3 } Variable & Input & Output \\
\hline Time (hour) & $\mathrm{X}$ & \\
Dry bulb temperature & $\mathrm{X}$ & \\
Wet bulb temperature & $\mathrm{X}$ & \\
Dew point temperature & $\mathrm{X}$ & \\
Relative humidity & $\mathrm{X}$ & \\
Wind speed & $\mathrm{X}$ & $\mathrm{X}$ \\
Wind direction & & \\
Total building power consumption & & \\
\hline
\end{tabular}

where $x_{i}$ is the normalized measurement, $x_{\min _{i}}$ the minimum of input parameter $i$ and $x_{\max _{i}}$ the maximum of input parameter $i$.

The input quantities are then passed on to the neighbouring hidden layer after application of a weight. A hidden layer totals up the weighted input received from each input neuron, associates it with a bias and then passes the result through a nonlinear transfer function (Figure 3). The final output layer collects all the quantities that were compressed through the activation function. The activation function used in this study was sigmoid function (Munakata, 1998). The purpose of a backpropagation algorithm is to minimize error, and to compute its associated optimal weight matrix. Error minimization process is explained in detail in Zurada (1992). At the beginning, all weights were assigned random values 


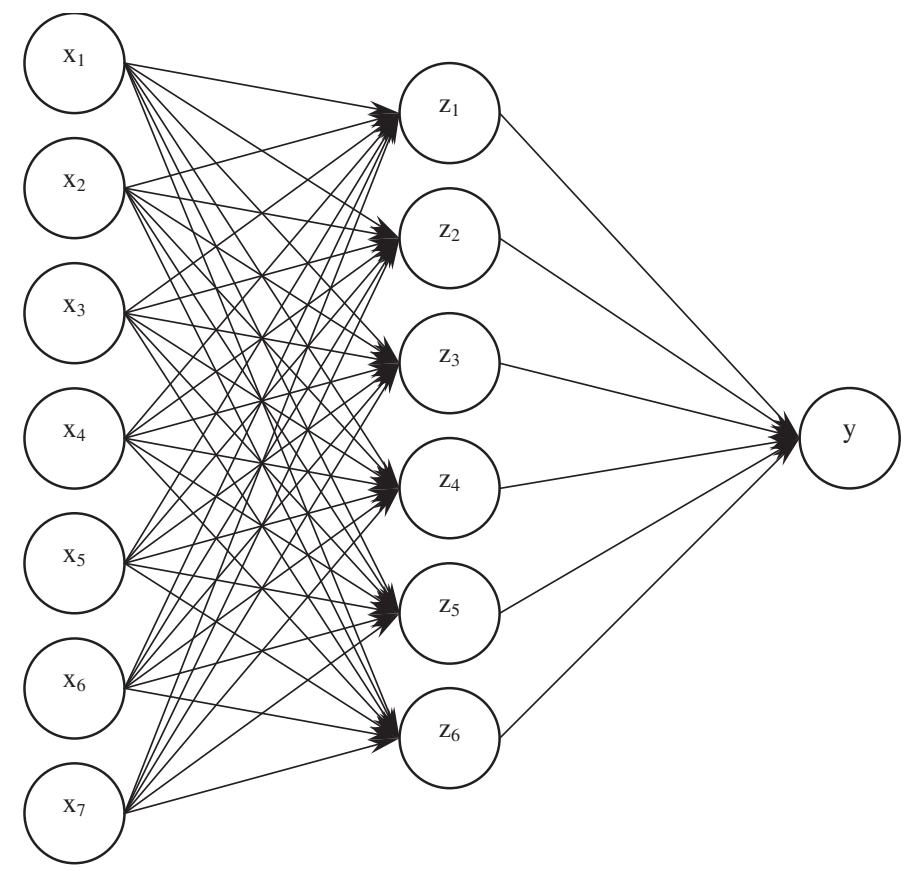

Figure 2. The ANN model architecture used in this study.

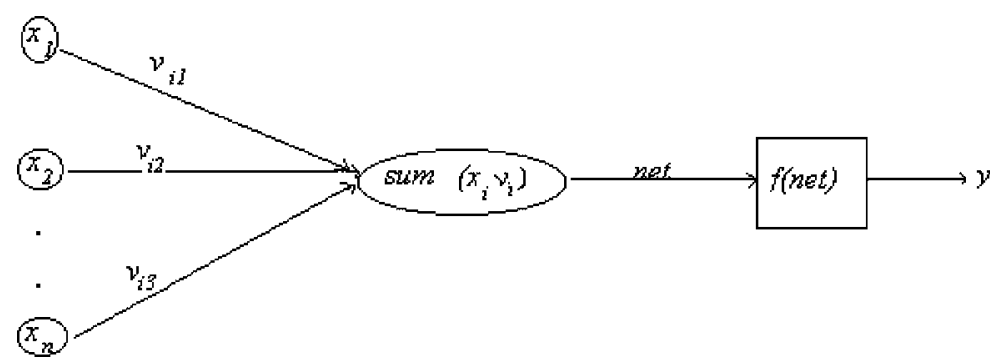

Figure 3. Activation function, $f($ net), compresses summed data.

between -1 and 1 . As the iteration continued, the weights were updated to approach their optimal values.

\section{MODEL CONSTRUCTION, APPLICATION AND RESULTS}

In this study, the common three layer-feedforward type of ANN was used. The input layer, hidden layer and the output layer contained 7, 6 and 1 neurons, respectively. Neurons in each layer are completely connected to each neuron in the neighbouring layer. No bias or momentum term was used in the generation of the model. The original data were composed of 121 sets each 
with 7 input parameters $\left(x_{1}, x_{2}, x_{3}, \ldots, x_{7}\right)$, and 1 output parameter $(y)$. The input quantities and their ranges are given in Table II. The data were first split into two parts to use for training ( 80 sets) and testing (41 sets) of the model. 2000 iterations were performed in this study to train the model. As shown in Figure 4, the resulting average absolute percent training error was $9.7 \%$ for the output variable of the total central plant power. The next stage of modelling involved testing, during which 41 measured data sets were fed to the optimized model to produce the model output for comparison to the physically measured data. The results indicated that the average testing errors were $10.0 \%$ for the total chiller power. The model was, therefore, successfully created, and was able to closely agree with the actual measured data (Figure 5). Parameters like the model architecture, the number of hidden layer neurons, the type of activation function and the scaling interval were selected as specified above after several trials with other parameter combinations. In most studies in the literature, the number of hidden layer neurons is about the same as the number of input layer neurons (Zurada, 1992; Munakata, 1998). In this study, a computer program that utilizes the ANN BP algorithm was written, and run in Matlab on a PC.

Table II. The data used in ANN model construction.

\begin{tabular}{llccc}
\hline & \multicolumn{1}{c}{ Variable } & \multicolumn{2}{c}{ Data used in model building } \\
\cline { 3 - 5 } Code & Minimum & Average & Maximum \\
\hline$x_{1}$ & Time $(53$ min after the hour) & 1 & 12.69 & 24 \\
$x_{2}$ & Dry bulb temperature $\left({ }^{\circ} \mathrm{C}\right)$ & 21.1 & 24.22 & 28.3 \\
$x_{3}$ & Wet bulb temperature $\left({ }^{\circ} \mathrm{C}\right)$ & 19.3 & 20.75 & 22.6 \\
$x_{4}$ & Dew point temperature $\left({ }^{\circ} \mathrm{C}\right)$ & 17.2 & 18.87 & 20 \\
$x_{5}$ & Relative humidity $(\%)$ & 56 & 72.82 & 90 \\
$x_{6}$ & Wind speed & 0 & 10.89 & 22 \\
$x_{7}$ & Wind direction & 0 & 66.36 & 360 \\
$y$ & Total chiller plant power $(\mathrm{kW})$ & 126.49 & 257.47 & 450.94 \\
\hline
\end{tabular}

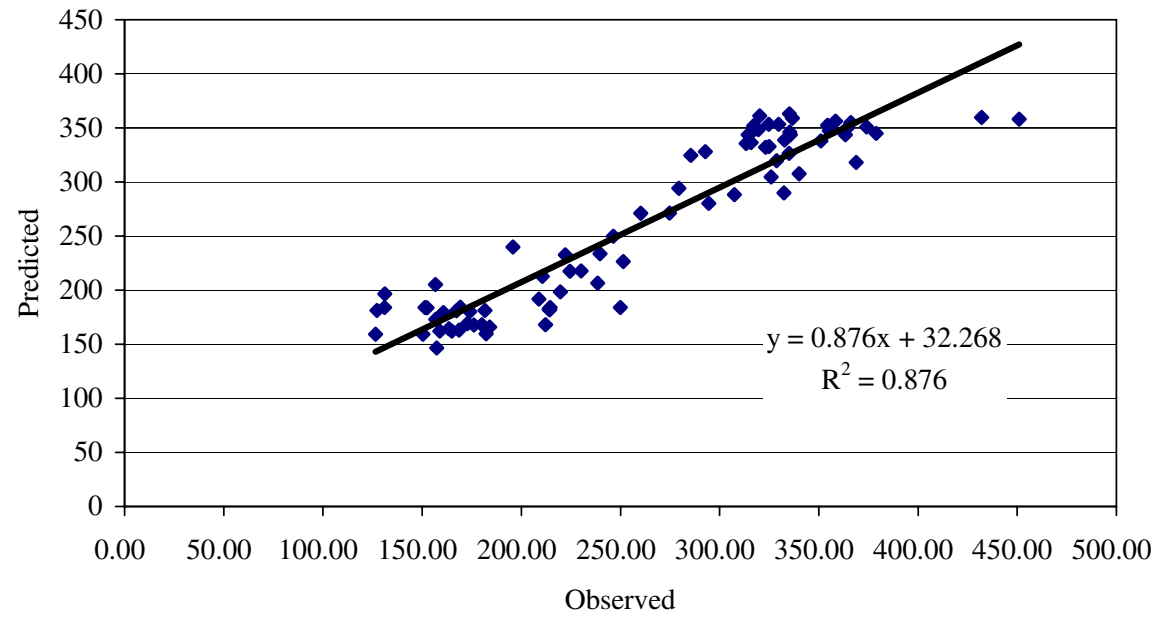

Figure 4. ANN model training results. 


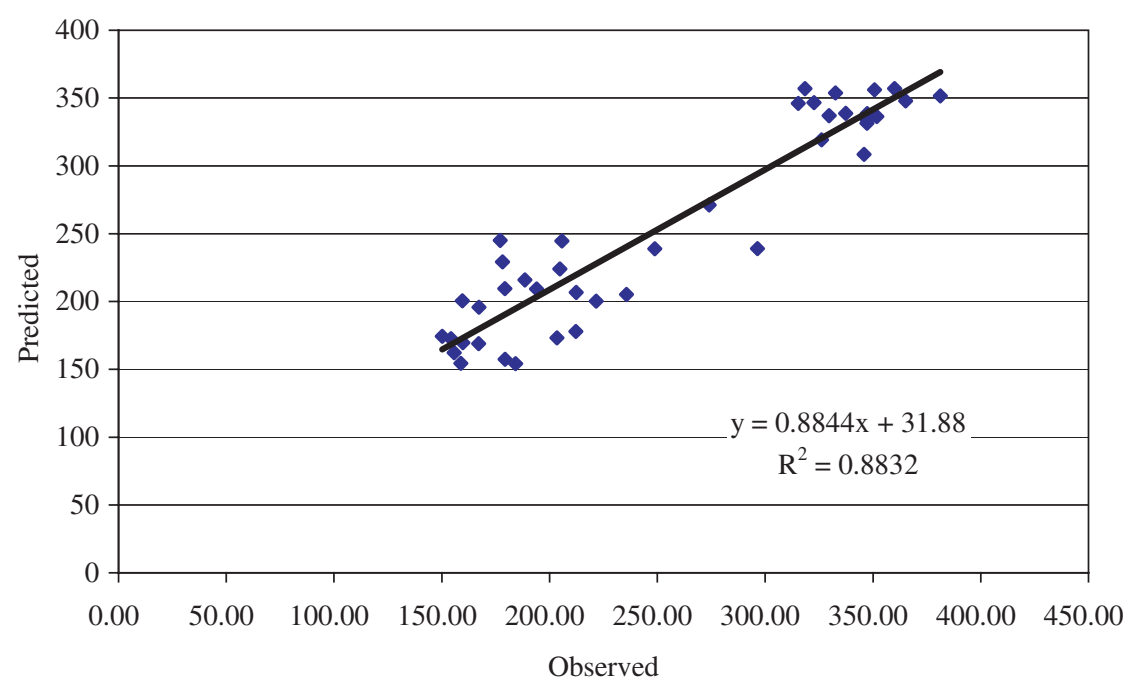

Figure 5. ANN model testing results.

\section{DISCUSSION}

The ANN model developed in this study processes data from two main origins: climate and HVAC system. Therefore, with the help of this model, we are able to predict the chiller plant power consumption as a function of meteorological parameters like the wind speed, wind direction, dry bulb and wet bulb temperatures and relative humidity. Air conditioning processes are controlled within narrow parameter ranges in Hawaii because the annual climate variation is narrow. Hence, the data range employed in this model construction was narrow. This has resulted in a model that can predict within a narrow range. The unique climate consistency in Hawaii indicates that there is a greater chance that the ANN methods can be successfully implemented in building energy predictions.

\section{CONCLUSIONS}

ANNs are useful tools for building energy analysis. Once an accurate ANN model is developed, a building engineer can easily apply this method to predict and evaluate a particular building energy performance without needing detailed knowledge of the ANN method. However, a generalized ANN method that can be used for building energy predictions with maximum success has yet to be developed. Additional factors such as data noise elimination, identification of the most suitable ANN architecture for a particular application, achieving lower coefficient of variations with consistency, have to be addressed before there can be full acceptance of the ANN methods in the building industry. A three-layer ANN model was developed as a case study for the prediction of total building chiller plant power consumption in a multipurpose high rise building. The satisfactory predictions of the observed chiller power by the model showed that ANN could be a very useful tool for the modelling of HVAC systems. The model 
lends itself to use by HVAC engineers as a guide for the conditions that give the lowest possible load when a sensitivity analysis is performed for the effects of the factors.

\section{ACKNOWLEDGEMENTS}

The authors would like to express their acknowledgement to Douglas Rauch from the Hawaiian Building Maintenance Company for providing building data for the case analysis presented in the paper.

\section{REFERENCES}

Ansett M, Kreider JF. 1993. Application of neural networking models to predict energy use. ASHRAE Transactions: Research 99(1):505-517.

Breekweg MRB, Gruber P, Ahmed O. 2000. Development of generalized neural network model to detect faults in building energy performance—part I, part II. ASHRAE Transactions: Research 4372:61-93.

Chonan Y, Nishida K, Matsumoto T. 1996. Great energy predictor shootout II: a Bayesian nonlinear regression with multiple hyper-parameters. ASHRAE Transactions: Symposia SA-96-3-1:405-411.

Cohen DA, Krarti M. 1995. A neural network modelling approach applied to energy conservation retrofits. Proceedings of Fourth International Conference on Building Simulation, Madison, WI, 423-430.

Curtiss PS. 1996. Examples of neural networks used for building system control and energy management. $A S H R A E$ Transactions: Symposia BN 97-16-1:909-913.

Curtiss PS, Kreider JF, Brandemuehl MJ. 1993. Energy management in central HVAC plants using neural networks. ASHRAE Transactions: Research 99(1):476-493.

Haberl JS, Thamilseran S. 1996. Predicting hourly building energy use: the great energy predictor shootout II: measuring retrofit savings—overview and discussion of results. ASHRAE Transactions 102(Pt. 2):419-435.

Hagan MT, Demuth HB, Beale MH. 1997. Neural Network Design. PWS Publishing Co: Boston, MA, USA.

Jang K-J, Bartlett EB, Nelson RM. 1996. Measuring retrofit energy savings using auto-associative networks. ASHRAE Transactions: Symposia SA-96-3-1:412-418.

Kreider JF, Claridge DE, Curtiss P, Haberl JS, Krarti M. 1995. Building energy use prediction and system identification using recurrent networks, transactions of the ASME. Journal of Solar Energy Engineering 117:161-166.

Munakata T. 1998. Fundamentals of the New Artificial Intelligence: Beyond Traditional Paradigms. Springer: New York.

Zurada MJ. 1992. Introduction to Artificial Neural Systems. PWS Publishing Company: Boston, MA, USA. 